\title{
Modified Kantrowitz Starting Criteria for Mixed Compression Supersonic Intakes
}

\author{
Andreas K. Flock* and Ali Gülhan \\ DLR, German Aerospace Center, 51147 Cologne, Germany
}

DOI: $10.2514 / 1 . J 057283$

\begin{abstract}
A proper understanding of the intake starting process of supersonic airbreathing engines is crucial for a successful operation of the supersonic aircraft, especially for three-dimensional geometries in which no reliable starting prediction for a broad Mach number range exists, and the widespread Kantrowitz criterion only provides a conservative prediction. Experimental investigations from the literature are reviewed and put into the perspective of the Kantrowitz theory. First, an empirical relation is developed that is valid for a wide Mach number range, and that can be calibrated to certain classes of intakes by the user. Second, the Kantrowitz assumptions are modified and a semiempirical relation is derived. The semiempirical relation turned out to be an optimistic limit for self-starting.
\end{abstract}

\section{Nomenclature}

$A$

$a$

$C_{1}$

$h$

$k$

M

$\dot{m}$

$p$

$T$

$\beta$

$\gamma$

$\theta$

$\Pi$

$\rho$

\section{Subscripts}

$i$

$=$ area, $\mathrm{m}^{2}$

$=$ speed of sound, $\mathrm{m} / \mathrm{s}$

$=$ constant

$=$ specific enthalpy, $\mathrm{J} / \mathrm{kg}$

$=$ constant

$=$ Mach number

$=$ mass flow, $\mathrm{kg} / \mathrm{s}$

$=$ pressure, $\mathrm{N} / \mathrm{m}^{2}$

$=$ temperature, $K$

$=$ shock angle, deg

$=$ ratio of specific heats

$=$ deflection angle, deg

$=$ pressure ratio

$=$ density, $\mathrm{kg} / \mathrm{m}^{3}$

$=$ internal portion

isentr. $\quad=$ isentropic

Kantr. $=$ Kantrowitz

$m=$ modified

$\max =$ maximum

poly. $\quad=$ polynomial

semiemp. $=$ semiempirical

, tot $=$ total

$=$ throat

$\infty \quad=$ freestream condition

Superscript

* $=$ chocked conditions

\section{Introduction}

$\mathbf{T}$ HE efficiency of hypersonic vehicles that fly within the lower region of the Earth's atmosphere can be increased by using

Received 9 March 2018; revision received 20 November 2018; accepted for publication 1 December 2018; published online Open Access 28 January 2019. Copyright $\odot 2018$ by Andreas K. Flock. Published by the American Institute of Aeronautics and Astronautics, Inc., with permission. All requests for copying and permission to reprint should be submitted to CCC at www.copyright.com; employ the eISSN 1533-385X to initiate your request. See also AIAA Rights and Permissions www.aiaa.org/randp.

*Research Scientist, Supersonic and Hypersonic Technologies Department, Institute of Aerodynamics and Flow Technology, Linder Höhe; andreas.flock@dlr.de.

'Department Head, Supersonic and Hypersonic Technologies Department, Institute of Aerodynamics and Flow Technology, Linder Höhe. airbreathing cycles instead of rocket engines. Because airbreathing cycles extract oxidizer from the surrounding atmosphere, they usually have a better propellant mass fraction and a higher specific impulse [1]. One airbreathing cycle particularly suited for the Mach number range between 5 and 12 is that of the supersonic combustion ramjet (scramjet) $[\underline{2}, \underline{3}]$.

In the current work, we focus on the air intake that serves as the compression system within the engine cycle. First, its purpose is to compress the incoming air flow to pressures and temperatures sufficient for combustion and to guide the flow into the combustion chamber. Second, because it serves as the interface between the freestream and the engine, almost all following components depend on a successful operation and proper understanding of the air intake.

The specific topic of the present paper is the process of establishing a supersonic flow throughout the intake, which is oftentimes referred to as intake starting. A not fully started intake usually results in multiple ramifications: First, due to subsonic flow through the intake, the mass flow and thus thrust of the engine both decrease. Second, the process of restarting the intake is followed by several subsequent difficulties such as reignition or reestablishment of a proper fuel flow rate, etc. Therefore, it is not only important to know when an intake will successfully start, it is likewise important to determine if the intake unstarts and restarts. Third, an intake unstart can jeopardize the maneuverability or stability of the entire vehicle if the thrust vector is not aligned with the center of mass or if, in a multiengine aircraft, only one intake unstarts. Finally, to assure intake starting over a broader flight envelope, additional components, such as variable geometry or bleed systems, might need to be added to the vehicle, which usually increase the weight and system complexity.

As we described in a previous study [4] for an arbitrary intake diffuser, there are two equations that relate the Mach number to the contraction ratio: First, isentropic compression to sonic flow at the intake throat limits the intake's contraction ratio to a maximum for a given Mach number:

$$
\mathrm{CR}_{\text {isentr. }}=\frac{A}{A^{*}}=\frac{1}{M}\left[\frac{2}{\gamma+1}\left(1+\frac{\gamma-1}{2} M^{2}\right)\right]^{(\gamma+1) /(2(\gamma-1))}
$$

Second, a normal shock covering the inflow plane of a quasi-onedimensional intake flow is swallowed when the following holds. In a converging duct, the subsonic portion downstream of the shock is accelerated. If the contraction is equal to or less than a contraction that would choke the flow, that is cause $M=1$ in the throat, the shock is swallowed. If the contraction is larger, the shock detaches and unstarts the flow. This second criterion is widely referred to as the

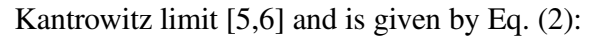

$$
\mathrm{CR}_{\text {Kantr. }}=\frac{A}{A^{*}}=\left[\frac{(\gamma+1) M^{2}}{(\gamma-1) M^{2}+2}\right]^{0.5}\left[\frac{(\gamma+1) M^{2}}{2 \gamma M^{2}-(\gamma-1)}\right]^{1 /(\gamma-1)}
$$


Intakes with an external compression overall contraction ratio and freestream Mach number are usually replaced by an internal contraction ratio and Mach number $M_{\mathrm{cl}}$ at the cowl closure location. The internal contraction ratio (CR) is defined by the area ratio of the cross-stream area at the cowl closure to the throat area:

$$
\mathrm{CR}_{i}=\frac{A_{\mathrm{cl}}}{A_{\infty}}
$$

Note that, oftentimes, it is difficult or impossible to measure $M_{\mathrm{cl}}$. Therefore, this parameter is frequently extracted from computational fluid dynamics (CFD) results by averaging the Mach number on the cross-stream area of the cowl closure location.

In Fig. 1, two means of plotting the isentropic and Kantrowitz limit are shown, along with empirical relations that will be explained later. Three regions are bounded by the two limits: first, a region in which intakes cannot work physically of $\mathrm{CR}>\mathrm{CR}_{\text {isentr. }}$; second, a region in which intakes are self-starting according to the Kantrowitz theory of $\mathrm{CR}<\mathrm{CR}_{\text {Kantr. }}$; and third, a critical region in between where intakes can work once successfully started.

From Fig. 1, it can be concluded that the two main parameters that influence intake starting are 1) the contraction ratio because, for a constant Mach number, intakes with low contraction are more likely to start than geometries with high contraction; and 2) the flight Mach number because, for a constant contraction ratio, intakes are more likely to start when the Mach number is increased (overspeeding [7]). Note that there is a limit in the contraction ratio for $M \rightarrow \infty$, which imposes a limit on the influence on the Mach number. For air and the constant ratio of specific heats of $\gamma=1.4$, this limit reads as follows:

$$
\lim _{M \rightarrow \infty} \mathrm{CR}_{\text {Kantr. }}=\left(\frac{(\gamma+1)}{(\gamma-1)}\right)^{0.5}\left(\frac{(\gamma+1)}{2 \gamma}\right)^{1 /(\gamma-1)}=1.666
$$

Other parameters such as the Reynolds number [8], the total freestream-to-wall temperature ratio [4]], or the boundary-layer thickness [9] were reported to have minor influences on intake starting.

The starting characteristics of most two-dimensional intake configurations seem to be in accordance with the Kantrowitz limit [10]. Various authors, however, reported self-starting to occur within the critical region of Fig. 1 , especially for three-dimensional geometries with mixed compression. Furthermore, Smart [11], and Sun and Zhang [12] developed empirical relations, which were valid for a certain Mach number range, to better predict intake starting within the critical region. Another relation is Eq. (5), which was proposed by Mölder et al. [13], and which will be explained subsequently. In the present paper, various self-starting intake configurations are collected and put into the perspective of the Kantrowitz plot (Fig. 1). Furthermore, we derive another empirical relation that is valid over a broad Mach number range, and which can be calibrated to intersect the critical region of the Kantrowitz plot at different levels. Additionally, we modified the underlying assumptions of Kantrowitz [] ] and derived a semiempirical relation to predict intake starting by multiplying the isentropic limit by a variable total pressure ratio. The semiempirical limit turned out to be an optimistic measure for intake starting. Finally, as the semiempirical relation cannot be expressed in an analytical equation, we propose several ways to model it in closed form.

\section{Methods and Materials}

\section{A. Literature Review}

In the present section, we summarize experimental starting data extracted from the literature that fulfill the following criteria: First, we claimed that the intake had to be started off or restarted from a blocked condition after the tunnel flow was fully developed. This prevented any enhancements of the unsteady effects during windtunnel start and excluded any experiments conducted in shortduration facilities, such as shock or expansion tubes. Second, an estimate of the Mach number at the cowl closure location needed to be given. Third, intake configurations with boundary-layer bleed were excluded because they make a proper calculation of the internal contraction ratio difficult.

In a previous study, we performed starting experiments in a hypersonic blowdown wind tunnel, known as $\mathrm{H} 2 \mathrm{~K}$, at the German Aerospace Center (DLR) in Cologne with a three-dimensional intake model [4,14], and we complemented the analysis with CFD [15]. The model was equipped with a movable cowl, permitting variable internal contraction so that, during an experiment, the $\mathrm{CR}_{i}$ was slowly decreased until intake starting occurred. Different cowl geometries that influenced the amount of overboard spillage, as well as the cowl closure Mach number and angle of attack, were investigated. The Mach number at the cowl closure position was estimated from CFD.

Various starting experiments were performed by Smart [16], Smart and Trexler [17], and Smart and Tetlow [18] on three-dimensional intakes with rectangular-to-elliptical shape transition (known as REST intakes) [16-18]. Tests were performed in blowdown wind tunnels down to $\bar{M}$ ach numbers of four, and mostly fixed geometry intakes were investigated. The Mach number at the cowl closure position was estimated from CFD. An intake was determined to be self-starting once it restarted after being blocked to an unstart with a throttle attached to the intake exit. For the low-Mach-number tests (Mach 4), the intake started only by the addition of bleed holes on the cowl side and sidewalls [17]. For the Mach 12 intake configuration [18], we estimated the Mach number at the cowl closure position to be equal to the Mach number for the geometry investigated earlier [16].

During a test campaign in a blowdown wind tunnel at the Virginia Polytechnic Institute and State University, Jacobsen et al. investigated the starting behavior of a streamline traced Busemann intake [19]. The model was equipped with a movable cowl and, after tunnel start, the internal contraction was decreased until starting occurred. During the Mach 4 experiments, Jacobsen et al. estimated the Mach number at the cowl closure position to be three.

Goldberg and Hefner performed starting experiments in a Mach 6 blowdown wind tunnel with a two-dimensional model without external compression [9]. Thus, the Mach number at the cowl closure
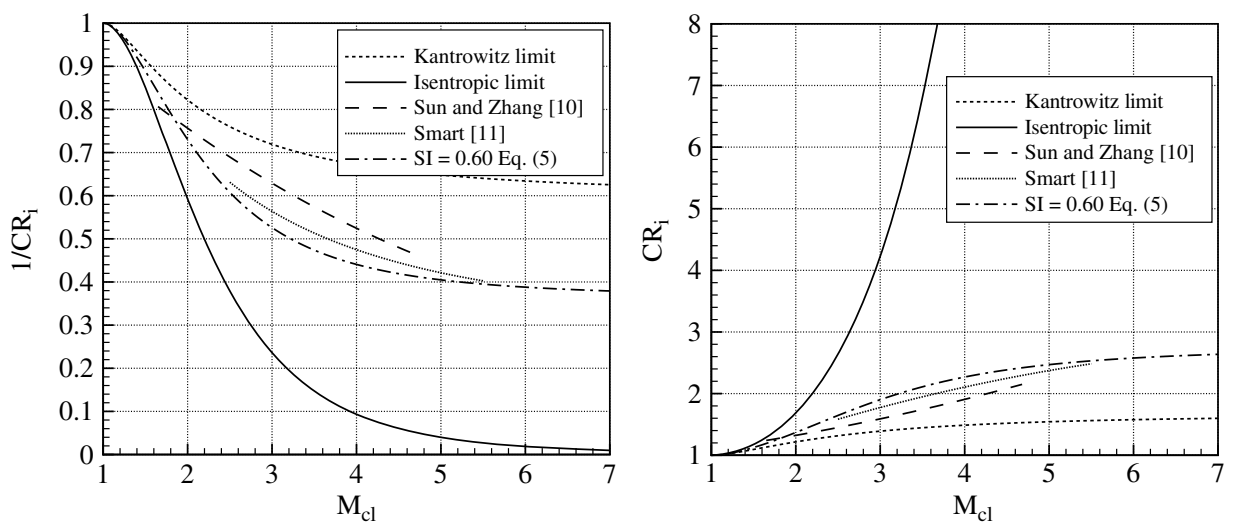

Fig. 1 Kantrowitz diagram, plotted in two different ways, along with the isentropic limit and empirical relations. 
was approximately equal to the freestream Mach number, and the flow entered the internal portion with a relatively thick boundary layer. The intake cowl could be rotated to modify the internal contraction, and flaps at the cowl's trailing edge were used to block off the flow and determine the self-starting capabilities of the intake. Among others, the influence of the Reynolds number and leadingedge radius were investigated.

In a blowdown wind tunnel, Emami et al. [20] investigated the unstart and restart qualities of a two-dimensional intake model with a simple ramp as external compression. Together with the freestream Mach number (with a value of six) and the deflection angle (11 deg), the Mach number at the cowl closure could be estimated. The intake cowl could be rotated around the throat location, and thus the internal contraction ratio could be varied. Once the intake unstarted, the restart position (and therefore the restart internal contraction) could be detected by opening the intake cowl. They observed that, with thinner boundary layers entering the internal portion of the intake, intake starting could be improved.

Finally, Mölder et al. [13] introduced the startability index (SI), which allows for linear interpolation in between the Kantrowitz and isentropic limits, and which can be calculated by the following:

$$
\mathrm{SI}=\frac{1 / \mathrm{CR}_{i}-1 / \mathrm{CR}_{\text {isentr. }}}{1 / \mathrm{CR}_{\text {Kantr. }}-1 / \mathrm{CR}_{\text {isentr. }}}
$$

They used the SI to characterize and group intakes, which started due to mass extraction. Two-dimensional Prandtl-Meyer intakes, for example, have improved starting characteristics due to overboard spillage. They derived that $\mathrm{SI}=0.6$ predicted the starting behavior of such two-dimensional intakes reasonably well, and they validated their results with inviscid Euler simulations.

\section{B. Empirical Relation}

Similar to the relations by Smart [11], and Sun and Zhang [12], we propose another criterion to predict intake starting. It should exhibit a Mach number independency for large Mach numbers and allow for fine-tuning by the user. Therefore, the requirements are summarized as follows:

1) For $M \rightarrow 1$, the contraction ratio should approach one.

2) For $M \rightarrow \infty$, the contraction ratio should approach a constant value (greater than 1.666).

3) The contraction ratio should be larger than the values from the Kantrowitz criterion but lower than the maximum contraction ratios for isentropic flow.

4) The relation should be variable to allow for an adjustment of the equation to different levels.

After incorporating these requirements, we came up with the following expression in which $C_{1}$ denotes a constant:

$$
\mathrm{CR}_{\text {empirical }}=\frac{\mathrm{CR}_{\text {Kantr. }}}{C_{1}+\mathrm{CR}_{\text {Kantr. }}\left(1-C_{1}\right)}
$$

The expression contains the Kantrowitz limit, and therefore results in a line that is similar in shape. The constant $C_{1}$ leads to different threshold values for $M_{\mathrm{cl}} \rightarrow \infty$. In Fig. 2, the empirical relation is

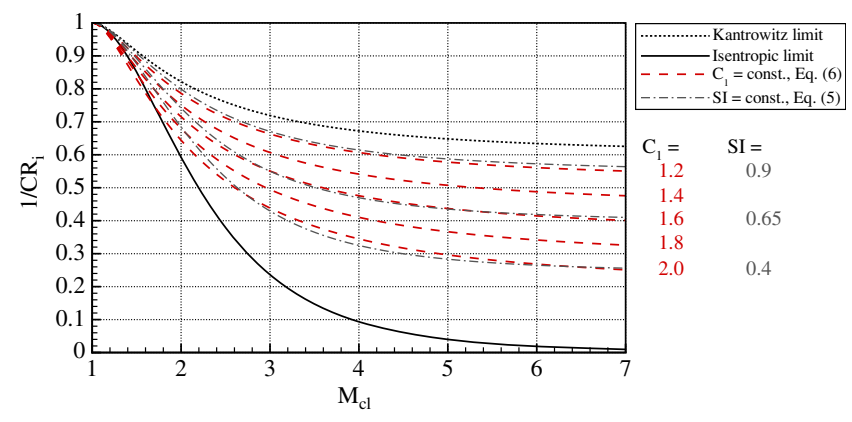

Fig. 2 Kantrowitz plot with empirical starting relation that allows adjustment via $C_{1}$ constant. plotted for different constants in the Kantrowitz plot. It can be seen that, for $C_{1}=1$, the empirical relation merges into the Kantrowitz line and, for increasing $C_{1}$, it stretches into the critical region. At low Mach numbers and with increasing $C_{1}, \mathrm{CR}_{\text {empirical }}$ intersects the isentropic relation, which makes Eq. (6) invalid. For a constant of $C_{1}=1.5$, for example, the empirical relation is invalid for a Mach number range of $1<M<1.32$. Therefore, low-Mach-number cases should be observed carefully when applying this relation. As will be shown when plotting the results of the Literature Review section (Sec. II.A), there are upper limits for the internal contraction ratios for self-starting. Those upper limits did not exceed Eq. (6) for $C_{1}=2$. We therefore propose to limit the constant to $1<C_{1}<2$. Note that a usage of $C_{1}>2.5$ would lead to a contraction ratio lower than zero for $M \rightarrow \infty$, which is not physical. The shape of the empirical relation is generally similar to the SI approach by Mölder et al. (Eq. (5) [13]). With a decreasing SI, their weighting of the isentropic limit increases. Therefore, our proposed empirical limit and their relation differ more strongly when approaching the isentropic line.

If the self-starting limits for one configuration are known at different Mach numbers, either by experiment or CFD, the empirical relation can be fitted to the data by adjusting $C_{1}$. Subsequently, the fit can be used to interpolate for data points at unknown Mach numbers. When varying other parameters, such as angle of attack, the selfstarting characteristics usually shift to larger or lower contraction ratios as the Mach number near the cowl closure changes (see discussion in Ref. [4]). When the starting characteristics at one Mach number are known for different angles of attack, the constant $C_{1}$ can be used to shift Eq. (6) to contraction ratios that match the results for these different angles of attack. This way, the starting characteristics at various angles of attack and other Mach numbers could be estimated. Thus, by varying $C_{1}$, the empirical approach allows for flexible adjustment of the starting limit to certain configurations.

\section{Semiempirical Relation}

We revisited the problem of predicting intake starting from the physical perspective with Kantrowitz theory as the starting position. As mentioned in the introduction, the theory is a conservative limit for intake starting, especially for three-dimensional flow configurations with mixed compression. Nevertheless, the slope of the Kantrowitz line resembles the general trends, which are an increasing $\mathrm{CR}$ with a larger Mach number and a Mach number independence for $M \rightarrow \infty$, correctly. Because the deficit of the Kantrowitz theory is mostly for three-dimensional cases, the initial assumption of one-dimensional flow has to be reconsidered. Furthermore, we argue that the assumption of a normal shock in front of the intake is less realistic in three-dimensional flows and that a rather strong oblique or curved shock is present and needs to be swallowed by the intake for starting. This is supported by Fig. $\underline{3}$, which shows schlieren images of unstarted (left) and started (right) intake flows, which were acquired during a previous study on intake starting [4]. For the started condition, the ramp shock is clearly visible, and no strong shock with a subsonic downstream portion is present. In the unstarted flowfield, however, a strong shock that is oblique is present and interacts with the ramp shock, therefore causing an increased spillage air flow across the intake cowl. Furthermore, an estimate of the extension of the oblique shock into the internal flowpath is drawn, with a separation zone in front of the entrance of the fully enclosed intake portion.

With the preceding discussion, the following reasoning was made: Fig. 4 shows flow through schematic diffusers with differing total pressure levels. In Fig. 4 (left), the inflow condition is unchanged. In Fig. 4 (middle and right), the inflow conditions have reduced the total pressure due to normal and oblique shocks, respectively. The flow in Fig. 4 (right) with the shock angle $\beta$ is roughly comparable to the unstarted condition in Fig. 3 . The flow in the throat section is always choked, which is indicated by the asterisk. In the Kantrowitz case (Fig. 4, middle) this total pressure reduction is due to the shock losses across the normal shock. It is important to note that the flows through the diffusers are regarded isentropic, and that the only source of reduced total pressure is due to the shocks. Furthermore, it is 

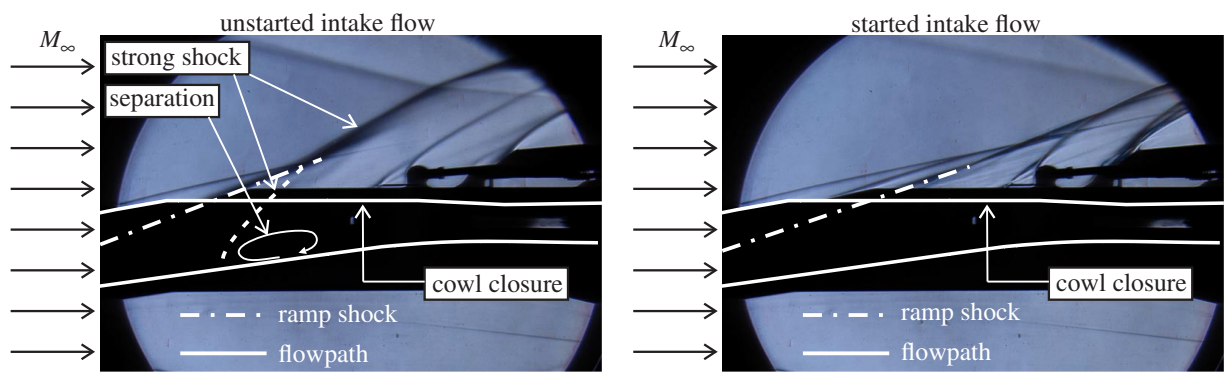

Fig. 3 Schlieren images of unstarted (left) and started (right) intake flows along with sketched shock positions and possible separation region in internal flowpath.

fully isentropic flow

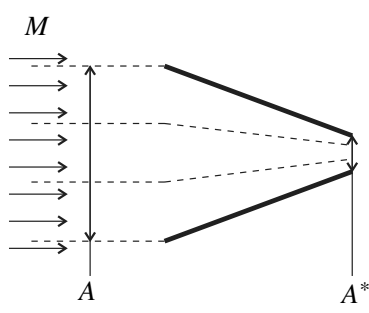

flow with normal shock

(Kantrowitz case)

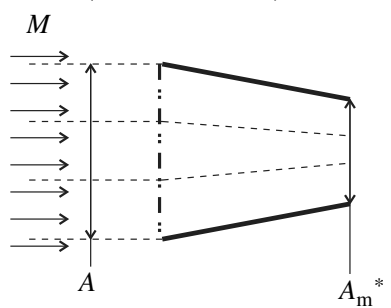

flow with oblique shock (semiempirical case)

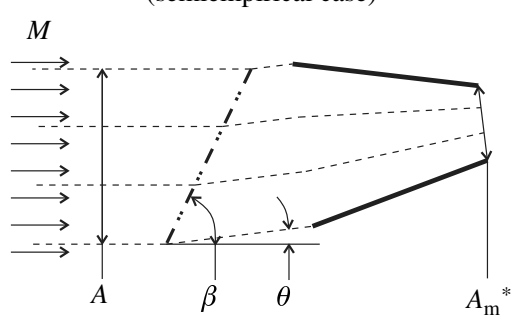

diffuser wall

$-\cdots-\cdots$ shock wave

Fig. 4 Sketch of diffuser flow; inflow conditions have no reduced total pressure (left), reduced total pressure due to normal shock (middle), and reduced total pressure due to oblique shock (right).

argued that the process of total pressure loss was adiabatic and no mass was lost, and thus $h_{t}=$ const. and $\dot{m}=$ const. for the two configurations.

Therefore, for the diffuser throat, at which the Mach number is one, the following holds:

$$
\rho^{*} A^{*} a^{*}=\rho_{m}^{*} A_{m}^{*} a_{m}^{*}
$$

With the assumption of an adiabatic process, it can be shown that the temperatures, and thus local speeds of sound, at the diffuser throat are equal:

$$
h_{t}=\text { const. } \Rightarrow T^{*}\left(1+\frac{\gamma-1}{2} M^{* 2}\right)=T_{m}^{*}\left(1+\frac{\gamma-1}{2} M_{m}^{* 2}\right)
$$

Thus, the following equation relates the throat area ratio to the density and the pressure ratio via the equation of state:

$$
\frac{A^{*}}{A_{m}^{*}}=\frac{\rho_{m}^{*}}{\rho^{*}}=\frac{p_{m}^{*}}{p^{*}}
$$

Total pressure is calculated via the following:

$$
p_{t}=p\left(1+\frac{\gamma-1}{2} M^{2}\right)^{\gamma /(\gamma-1)}
$$

And, because the Mach number at the diffuser throat is one, the total and static pressures can be related for the two diffuser configurations:

$$
\frac{p_{m}^{*}}{p^{*}}=\frac{p_{t, m}^{*}}{p_{t}^{*}}
$$

Together with Eq. (9), this yields an expression that relates the two throat areas to their difference in freestream total pressure:

$$
\frac{A^{*}}{A_{m}^{*}}=\frac{p_{t, m}^{*}}{p_{t}^{*}}
$$

This expression proves that the diffuser with reduced total pressure chokes earlier. Furthermore, it can be modified to relate the contraction ratios of the two configurations and the total pressure loss:

$$
\frac{A}{A_{m}^{*}}=\frac{A}{A^{*}} \frac{p_{t, m}^{*}}{p_{t}^{*}}
$$

Thus, for the assumption of a constant total pressure loss, the isentropic limit can simply be modified via the following:

$$
\mathrm{CR}_{m}=\mathrm{CR}_{\text {isentr. }} \times \Pi_{t}
$$

The total pressure loss is given by $\left(1-\Pi_{t}\right)$. Note that the preceding derivation can also be found in the related research area of shock swallowing within supersonic wind tunnels ([21] p. 34). With Eq. (14), the lines of constant total pressure can be added to the Kantrowitz diagram (Fig. 5). The intersections between the Kantrowitz line and the lines of constant total pressure correspond to the total pressure loss due to a normal shock, which increases with

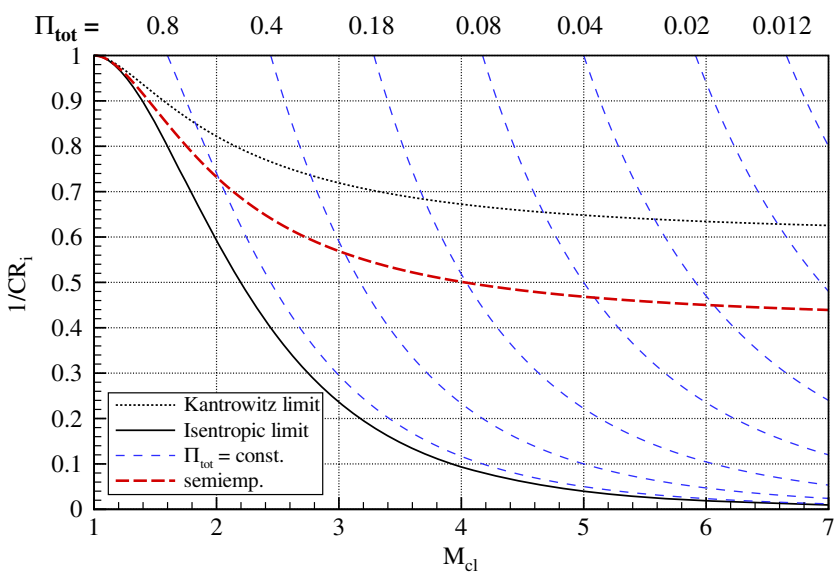

Fig. 5 Kantrowitz plot with lines of constant total pressure and semiempirical limit. 


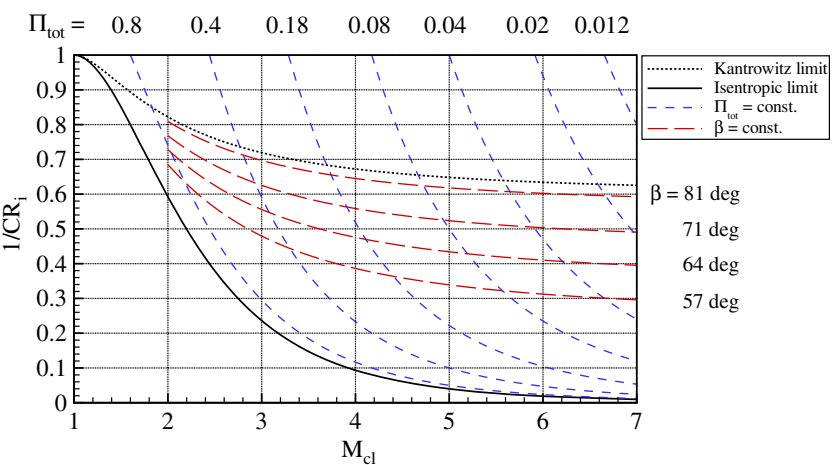

Fig. 6 Kantrowitz plot with lines of constant total pressure and constant shock wave angle.

Mach number. Lines within the critical region correspond to flow that did experience less total pressure loss, whereas the isentropic line resembles the limit for $\Pi_{t} \rightarrow 1$. The lines of constant total pressure loss for which $\mathrm{CR}_{m}<\mathrm{CR}_{\text {Kantr. }}$ holds denote cases in which the total pressure loss exceeded the losses due to a normal shock: for example, by additional viscous losses in the boundary layer.

Let us now consider that the shock that is swallowed during intake starting is not a normal, but rather oblique shock, such as depicted schematically in Fig. 3. Furthermore, while being swallowed for intake starting, the shock has to be somehow attached to the closing of the intake cowl. Then, in the most conservative case, the shock angle would be $\beta_{\max }$, which is the largest oblique shock angle that can still be attached, and which would vary with Mach number. The following equation relates the Mach number, the deflection angle $\theta$, and the shock angle:

$$
\tan (\theta)=2 \cot (\beta) \frac{M_{1}^{2}(\sin (\beta))^{2}-1}{M_{1}^{2}(\gamma+\cos (2 \beta))+2}
$$

To calculate $\beta_{\max }$, for one Mach number, Eq. (15) has to be solved for the largest $\theta$ to occur numerically. With $\beta_{\max }$ being a function of the Mach number and maximum deflection angle $\theta_{\max }$, a total pressure loss can be calculated, with which a modified starting limit is obtained:

$$
\mathrm{CR}_{\text {semiemp. }}=\mathrm{CR}_{\text {isentr. }} \times \Pi_{t}\left(\beta_{\max }\left(\theta_{\max }, M\right)\right)
$$

Note that $\beta_{\max }$ is not the largest shock angle to occur but is rather the shock angle related to the highest deflection possible $\theta_{\max }$ before an oblique shock detaches. Equation (16) is referred to as the semiempirical limit, and it is plotted in Fig. 5.

Finally, another mean to characterize the critical region can be derived when using a constant shock angle to determine the total pressure loss. The resulting lines of the constant shock angle are plotted in Fig. 6. Analogously, the intersections between the lines of the constant shock angle and the lines of the constant total pressure represent the corresponding total pressure loss at the respective Mach number. The behavior of the lines of constant shock angle is similar to the empirical relation that could be calibrated via the constant $C_{1}$ [see Eq. (6)]. Note that the shock wave angle is limited by the Mach angle $\left[\mu=\sin ^{-1}(1 / M)\right]$, which needs to be considered at low Mach numbers and low shock wave angles when constructing the relation.

\section{Results}

All self-starting configurations reviewed in Sec. II.A are plotted in Fig. 7 along with the semiempirical, Kantrowitz, isentropic, and empirical limits from the literature. The unstarted configurations are shown as unfilled symbols. All contraction ratios fell into the critical region and emphasize the conservative nature of the Kantrowitz theory. The semiempirical relation gives a better estimate for starting when compared to the intake configurations that were self-starting for high contraction ratios. According to the experimental data points, the semiempirical limit is located at the critical end at which intakes are self-starting. A reason for this could be that viscous effects

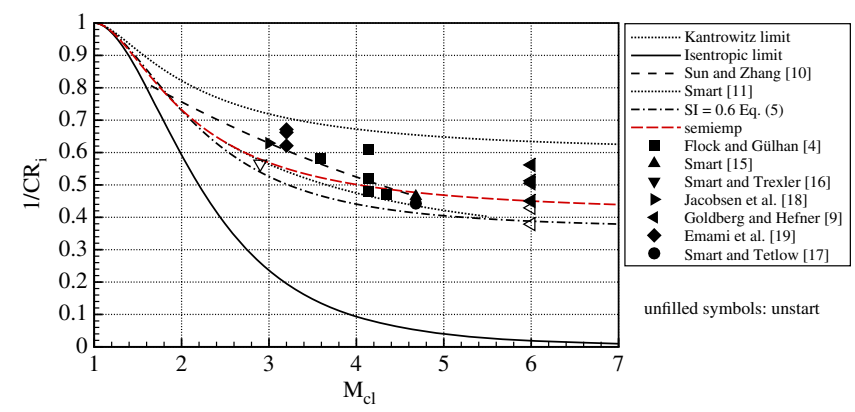

Fig. 7 Self-developed semiempirical relation in comparison with results on intake starting obtained from the literature.

downgrade the achievable internal contraction ratio additionally for self-starting. Therefore, the semiempirical limit can be regarded as an optimistic measure.

Compared to Smart's limit [11], the semiempirical relation is comparable for $M<3.5$ and becomes more conservative for higher Mach numbers. Compared to Sun and Zhang's limit [12], the semiempirical relation is slightly more optimistic. Compared to a starting index of 0.6 (Eq. (5) by Mölder et al. [13]), the two relations are similar at low Mach numbers; their differences increase with Mach number, whereas Eq. (5) yields larger contraction ratios. Overall, the semiempirical relation is valid for the entire Mach number range and correctly predicted the high Mach number results by Goldberg and Hefner [9].

It is impossible to give an analytical equation for the semiempirical limit in closed form. To provide a practical engineering method, we propose four options to quickly approximate Eq. (16). Note that all options are based on reproducing the shape of the curve of the semiempirical limit as closely as possible.

1) First, it can be represented when using the empirical relation in Eq. (6) with a constant of $C_{1}=1.5$.

2) Second, a constant oblique shock angle of $66.7 \mathrm{deg}$ can be used in Eq. (15) to determine a total pressure loss that will be similar to the one in the semiempirical relation.

3) Third, a polynomial fit $\mathrm{CR}_{\text {poly. }}$ to the semiempirical relation is given by the following:

$$
\mathrm{CR}_{\text {poly. }}=\left(k_{0}+k_{1} M_{\mathrm{cl}}^{1}+k_{2} M_{\mathrm{cl}}^{2}+k_{3} M_{\mathrm{cl}}^{3}+k_{4} M_{\mathrm{cl}}^{4}+k_{5} M_{\mathrm{cl}}^{5}\right)^{-1}
$$

with the constants

$$
\begin{gathered}
k_{0}=1.6244 \\
k_{1}=-7.4293 \times 10^{-1} \\
k_{2}=1.9331 \times 10^{-1} \\
k_{3}=-2.5456 \times 10^{-2} \\
k_{4}=1.6652 \times 10^{-3} \\
k_{5}=-4.2966 \times 10^{-5}
\end{gathered}
$$

4) Fourth, using the startability index proposed by Mölder et al. (see Eq. (5) and [13]) with SI $=0.685$ also duplicates the semiempirical limit.

The first three approximate expressions have to be applied carefully when approaching Mach 1 . The differences between Eq. (16) and the proposed approximations are quantified in Fig. $\underline{8}$. They were calculated by subtracting and then dividing by the contraction ratio of the semiempirical limit. All approximations were within $\pm 3 \%$ of the semiempirical limit; the empirical limit with $C_{1}=1.5$ and the polynomial fit were even within $\pm 2 \%$. For Mach 


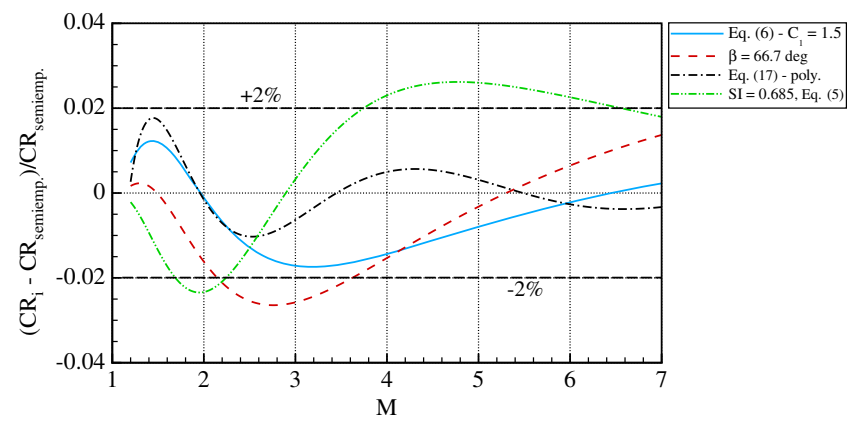

Fig. 8 Differences between the semiempirical relation and the four approximations.

numbers greater two, the polynomial fit was within $\pm 1 \%$ of the semiempirical value.

\section{Conclusions}

In the present paper, literature about intake starting to collect configurations with self-starting capability was reviewed. The data were put into the perspective of the Kantrowitz theory and were within critical limits in which starting should not occur according to Kantrowitz [6]. An empirical relation was derived that can be varied by the user via a constant. Furthermore, the assumptions made by Kantrowitz were modified to derive a semiempirical relation. Thereto, the isentropic limit was multiplied with a variable total pressure ratio that depended on Mach number. The main findings include the following:

1) The semiempirical relation was an optimistic limit to the intake starting data from the literature. As it cannot be given in one analytical equation, several approximations were proposed, which were at least within $\pm 3 \%$ of the semiempirical limit.

2) The empirical relation could be set to be similar to the semiempirical relation, or it could be adjusted to certain classes of intakes, as explained in Sec. II.B.

3 ) Both relations generally resemble the slope of the Kantrowitz line and are valid over a broader Mach number range than other empirical relations.

\section{Acknowledgments}

The present work was partly funded during the late phase of Research Training Group 1095/2 (2005-2014). Furthermore, the research was part of the first author's doctoral dissertation.

\section{References}

[1] Heiser, W. H., and Pratt, D. T., Hypersonic Airbreathing Propulsion, AIAA Education Series, AIAA, Washington, D.C., 1994, p. 110.

[2] Curran, E. T., "Scramjet Engines: The First Forty Years Introduction," Journal of Propulsion and Power, Vol. 17, No. 6, 2001, pp. 1138-1148. doi: $10.2514 / 2.5875$

[3] Smart, M. K., "Scramjets," Aeronautical Journal, Vol. 111, No. 1124, 2007, pp. 605-619. doi: $10.1017 / \mathrm{S} 0001924000004796$

[4] Flock, A. K., and Gülhan, A., "Experimental Investigation of the Starting Behavior of a Three-Dimensional Scramjet Intake," AIAA Journal, Vol. 53, No. 9, 2015, pp. 2686-2693. doi: $10.2514 / 1 . J 053786$
[5] Oswatitsch, K., "Pressure Recovery for Missiles with Reaction Propulsion at High Supersonic Speeds," NACA TR 1140, 1944.

[6] Kantrowitz, A., and Donaldson, C. d., "Preliminary Investigation of Supersonic Diffusers," NACA TR L5D20, 1945.

- [7] Timofeev, E. V., Tahir, R. B., and Mölder, S., "On Recent Developments Related to Flow Starting in Hypersonic Air Intakes," 15th AIAA International Space Planes and Hypersonic Systems and Technologies Conference, AIAA Paper 2008-2512, 2008, pp. 1-9. doi: $10.2514 / 6.2008-2512$

[8] Hohn, O. M., "Auslegung und Charakterisierung Eines Dreidimensionalen Scramjet-Einlaufs mit Hohem Verdichtungsverhältnis und Variabler Innenkontraktion," Ph.D. Thesis, Univ. of Stuttgart Stuttgart, Germany, 2014.

[9] Goldberg, T. J., and Hefner, J. N., "Starting Phenomena for Hypersonic Inlets with Thick Turbulent Boundary Layers at Mach 6," NASA TN D6280,1971

[10] Wie, D. M. V., Kwok, F. T., and Walsh, R. F., "Starting Characteristics of Supersonic Inlets," 32nd Joint Propulsion Conference, AIAA Paper 1996-2914, 1996 doi:10.2514/6.1996-2914

[11] Smart, M. K., "How Much Compression Should a Scramjet Inlet Do?" AIAA Journal, Vol. 50, No. 3, 2012, pp. 610-619. doi:10.2514/1.J051281

[12] Sun, B., and Zhang, K.-Y., "Empirical Equation for Self-Starting Limit of Supersonic Inlets," Journal of Propulsion and Power, Vol. 26, No. 4, 2010, pp. 874-875. doi:10.2514/1.46798

[13] Mölder, S., Timofeev, E. V., and Tahir, R. B., "Flow Starting in High Compression Hypersonic Air Inlets by Mass Spillage," 40th AIAA/ ASME/SAE/ASEE Joint Propulsion Conference and Exhibit, AIAA Paper 2004-4130, 2004, pp. 1-16. doi:10.2514/6.2004-4130

[14] Flock, A. K., "Design and Performance Analysis of Three-Dimensional Air Intakes for Supersonic Combustion Ramjet Engines," Ph.D. Thesis, DLR-FB-2017-01, Univ. of Stuttgart, Stuttgart, Germany, 2017.

-[15] Flock, A. K., and Gülhan, A., "Experimental and Numerical Performance Analysis of a Self-Starting Three-Dimensional Scramjet Intake," Journal of Propulsion and Power, Vol. 33, No. 6, 2017, pp. $1570-1580$. doi:10.2514/1.B36602

[16] Smart, M. K., "Experimental Testing of a Hypersonic Inlet with Rectangular-to-Elliptical Shape Transition," Journal of Propulsion and Power, Vol. 17, No. 2, 2001, pp. 276-283. doi: $10.2514 / 2.5774$

[17] Smart, M. K., and Trexler, C. A., "Mach 4 Performance of Hypersonic Inlet with Rectangular-to-Elliptical Shape Transition," Journal of Propulsion and Power, Vol. 20, No. 2, 2004, pp. 288-293. doi:10.2514/1.1296

[18] Smart, M. K., and Tetlow, M. R., "Orbital Delivery of Small Payloads Using Hypersonic Airbreathing Propulsion," Journal of Spacecraft and Rockets, Vol. 46, No. 1, 2009, pp. 117-125. doi:10.2514/1.38784

[19] Jacobsen, L. S., Tam, C.-J., Behdadnia, R., and Billig, F. S., "Starting and Operation of a Streamline-Traced Busemann Inlet at Mach 4," 42nd AIAA/ASME/SAE/ASEE Joint Propulsion Conference and Exhibit, AIAA Paper 2006-4508, 2006, pp. 1-19. doi: $10.2514 / 6.2006-4508$

[20] Emami, S., Trexler, C. A., Auslender, A. H., and Weidner, J. P., "Experimental Investigation of Inlet-Combustor Isolators for a DualMode Scramjet at a Mach Number of 4," NASA TP 3502, 1995.

[21] Hermann, R., Supersonic Inlet Diffusers and Introduction to Internal Aerodynamics, Minneapolis-Honeywell Regulator Co., Minneapolis, MN, 1956, p. 34.

Y. Zhou Associate Editor 